\title{
Symptomatische Kopfschmerzen
}

Das vorliegende Themenheft der Nervenheilkunde beschäftigt sich mit den symptomatischen Kopfschmerzen. Dieser Bereich wird in der Kopfschmerzforschung und -behandlung normalerweise nur am Rande abgehandelt. Dies liegt zum einen daran, dass die Kopfschmerzen häufig das Symptom einer behandelbaren Grunderkrankung sind und somit bei geeigneter Therapie oft vollständig abklingen. Zum anderen sind symptomatische Kopfschmerzen oft nur ein Symptom unter mehreren und führen häufig nicht zu einer ärztlichen Konsultation mit Kopfschmerzen als Leitsymptom. Dennoch gibt es Erkrankungen, bei denen Kopfschmerz das erste oder das führende Symptom ist und bei denen es therapeutisch auch in erster Linie um die Kontrolle des Kopfschmerzes und nicht um die Behandlung der Grunderkrankung geht.

Die Internationale Kopfschmerzklassifikation unterscheidet 8 (von 13) Kapitel mit symptomatischen Kopfschmerzen mit über 150 Unterarten, dennoch leiden nur unter 5\% der Patienten, die sich wegen Kopfschmerzen ärztlich vorstellen, an einem symptomatischen Kopfschmerz. Auch dieses Missverhältnis führt zu einer Vernachlässigung von symptomatischen Kopfschmerzen in der ärztlichen Praxis gleichzeitig vermuten Patienten selbst häufig einen symptomatischen Kopfschmerz als Ursache ihrer Beschwerden. Dabei sind manche symptomatische Kopfschmerzen gut zu behandeln, wenn auch gelegentlich schwer zu erkennen. Außerdem muss immer berücksichtigt werden, dass symptomatische Kopfschmerzen in ihrer Semiologie idiopathischen Kopfschmerzen sehr ähnlich (bis identisch) sein können. Auch dies muss der Kopfschmerzbehandler berücksichtigen.

Die Kriterien der Internationalen Kopfschmerzklassifikation haben früher verlangt, dass für die definitive Diagnose eines symptomatischen Kopfschmerzes eine erfolgreiche Therapie der Grunderkrankung auch zu einem Abklingen des Kopfschmerzes führen muss. Dies ist aber aus mehreren Gründen nicht immer sinnvoll und wurde deswegen in der nunmehr aktuellen
3. Auflage gestrichen. Es genügt nun, einen kausalen Zusammenhang zwischen der zugrunde liegenden Ursache und den Kopfschmerzen zu belegen. Dies kann auf verschiedenen Ebenen geschehen, nicht nur durch den Wegfall der Ursache. Mit der Systematik der Klassifikation von symptomatischen Kopfschmerzen setzt sich die Arbeit von Veit Mylius und Michael Teepker auseinander.

Die hier vorgestellten Arbeiten beziehen sich auf die häufigsten Aspekte der symptomatischen Kopfschmerzen. So sind Kopfschmerzen bei Liquordruckveränderungen häufig und leider auch oft verkannt. Hier bietet die Arbeit von Jan Hoffmann und Stefanie Förderreuther Hilfestellung. Weitere wichtige klinische Bilder sind der zervikogene Kopfschmerz, wie die Arbeit von Achim Frese und Kollegen zeigt, und der symptomatische Gesichtsschmerz, der von Andreas Gantenbein et al. diskutiert wird.

Einen besonders schwierigen Aspekt bei symptomatischen Kopfschmerzen stellen die psychogenen Kopfschmerzen dar. Hier wird landläufig von einem klaren kausalen Zusammenhang zwischen psychischen Beschwerdebildern und Kopfschmerzen ausgegangen. In der wissenschaftlichen Literatur findet sich dagegen kaum ein seriöser Beleg für diese Hypothese. Deswegen findet sich in der aktuellen Kopfschmerzklassifikation nur ein kurzer Abschnitt über Kopfschmerzen, die wirklich monokausal durch psychiatrische Erkrankungen verursacht werden. Mit diesem Thema setzt sich die Arbeit von Karsten Henkel auseinander.

Schließlich stellt auch die Koexistenz von idiopathischen und symptomatischen Ursachen für dieselbe Kopfschmerzsemiologie gelegentlich ein Problem im Alltag dar. So ist der Donnerschlagkopfschmerz für verschiedene Situationen beschrieben, wird aber eben auch als idiopathische Form klassifiziert. Hier gelingt eine $\mathrm{Ab}$ grenzung manchmal nicht sicher. Mit diesem Thema beschäftigt sich die Arbeit von Katharina Kamm und Koautoren. Ähnliches gilt für die trigemino-autonomen

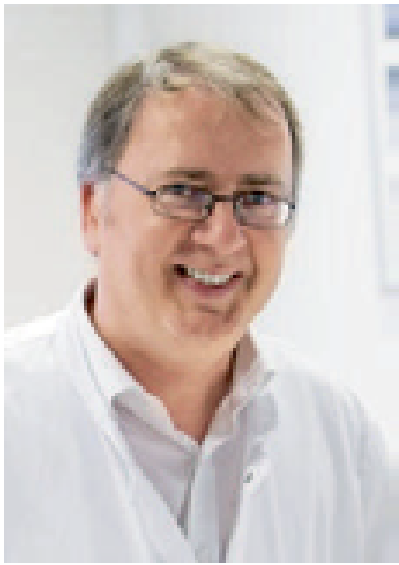

Prof. Dr. med. Dr. phil. Stefan Evers, Neurologische Klinik, Krankenhaus Lindenbrunn, Coppenbrügge

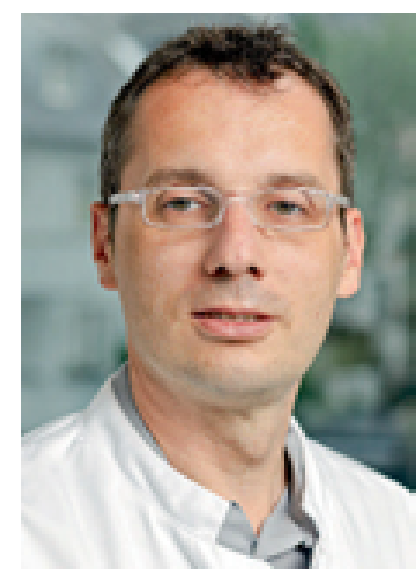

Priv.-Doz. Dr. med. Charly Gaul, Migräne- und Kopfschmerzklinik Königstein, Königstein im Taunus 
Kopfschmerzen, die ebenfalls selten mal eine symptomatische Ursache haben können, dies wird in der Arbeit von Charly Gaul und Dagny Holle-Lee dargestellt. Kopfschmerzen sind für viele Patienten Anlass, an einen Hirntumor zu denken. Dabei sind Kopfschmerzen bei Hirntumoren als Erstsymptom selten. Wann Kopfschmerzen bei Hirntumoren auftreten und wie sie therapiert werden können, darüber referiert Stefan Evers. Es ist ein durch die Internationale Kopfschmerzgesellschaft akzeptierter Konsens, dass die häufige Einnahme von Akutmedikation zur Zunahme oder Chronifizierung von episodischen Kopfschmerzen führen kann. Dieser Kopfschmerz wird als Medikamentenübergebrauchskopfschmerz bezeichnet. Die Übersichtsarbeit von Zaza Katsarava und Steffen Nägel beleuchten die Autoren den aktuellen Stand der Literatur zu dieser Form des Kopfschmerzes. Schließlich beschäftigt sich der Beitrag von Torsten Kraya und Martin Marziniak mit dem Zusammenhang zwischen vaskulären Erkrankungen und Kopfschmerzen. Während viel über die vaskulären Folgen der primären Kopfschmerzen wie z. B. der Migräne bekannt ist, ist umgekehrt noch wenig geklärt. Der Artikel schließt auch Daten einer aktuellen epidemiologischen Studie der DMKG mit ein.

Wir wünschen interessante Entdeckungen bei der Lektüre dieses Themenheftes und insgesamt eine größere Aufmerksamkeit für das Thema der symptomatischen Kopfschmerzen.

\section{Stefan Evers, Coppenbrügge}

Charly Gaul, Königstein im Taunus

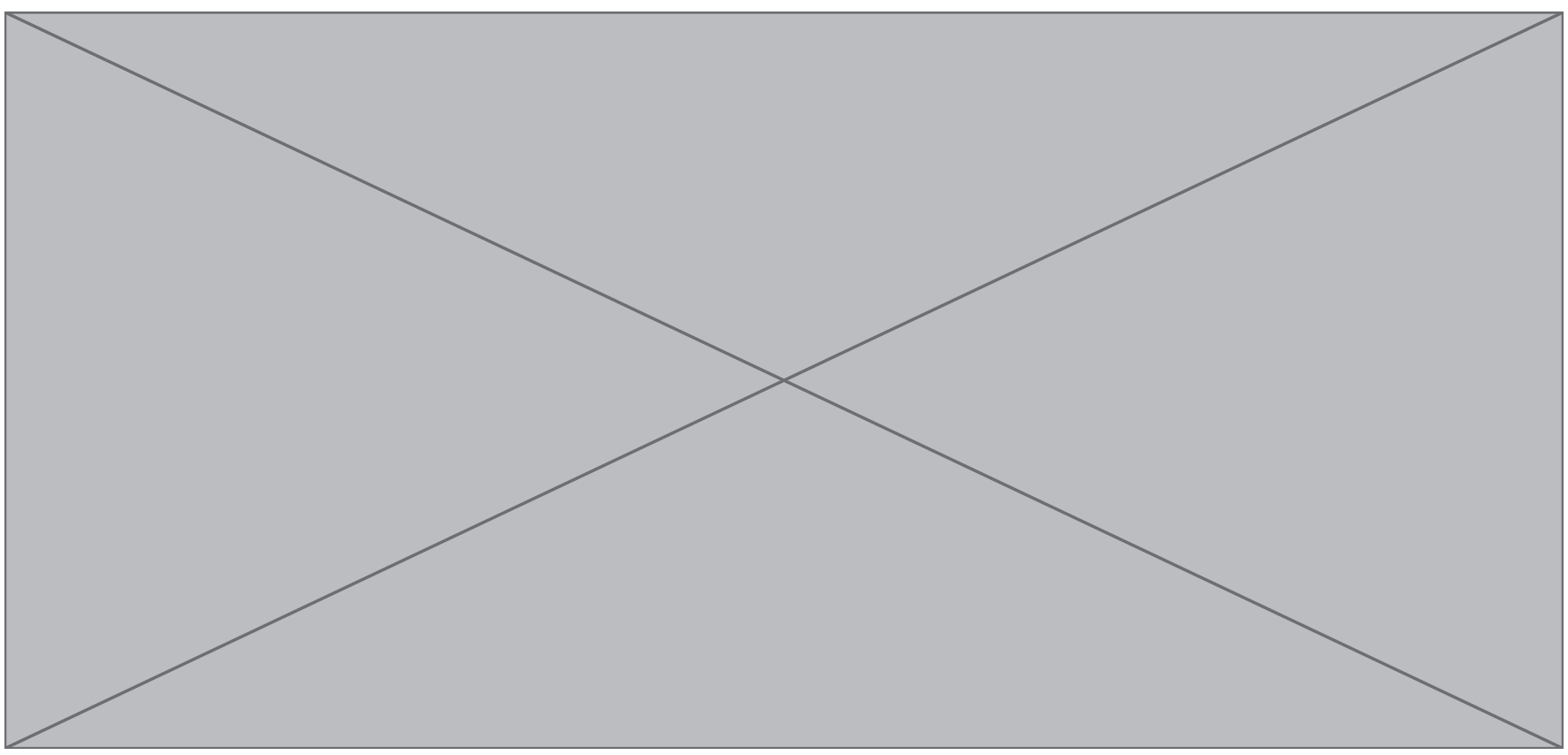

